\title{
RESEARCH ON AIRBORNE SAR IMAGING BASED ON ESC ALGORITHM
}

\author{
X. T. Dong ${ }^{\mathrm{ab}}$, X. J. Yue ${ }^{\mathrm{ac}}$, Y. H. Zhao ${ }^{\mathrm{ac}}$, C. M. Han ${ }^{\mathrm{ac} *}$ \\ ${ }^{a}$ Microwave System Engineering Division, Institute of Remote Sensing and Digital Earth, Chinese Academy of Sciences, Beijing, \\ 100094 China-(dongxt, yuexj, zhaoyh, hancm)@ radi.ac.cn \\ ${ }^{\mathrm{b}}$ University of Chinese Academy of Sciences, Beijing, 100049 China \\ ${ }^{c}$ Department of Airborne remote sensing, Sanya Institute of Remote Sensing, Sanya, 572029 China
}

SarCon 2017, WG III/3

KEY WORDS: SAR imaging, Motion errors, ECS, MOCO

\begin{abstract}
:
Due to the ability of flexible, accurate, and fast obtaining abundant information, airborne SAR is significant in the field of Earth Observation and many other applications. Optimally the flight paths are straight lines, but in reality it is not the case since some portion of deviation from the ideal path is impossible to avoid. A small disturbance from the ideal line will have a major effect on the signal phase, dramatically deteriorating the quality of SAR images and data. Therefore, to get accurate echo information and radar images, it is essential to measure and compensate for nonlinear motion of antenna trajectories. By means of compensating each flying trajectory to its reference track, MOCO method corrects linear phase error and quadratic phase error caused by nonlinear antenna trajectories. Position and Orientation System (POS) data is applied to acquiring accuracy motion attitudes and spatial positions of antenna phase centre (APC). In this paper, extend chirp scaling algorithm (ECS) is used to deal with echo data of airborne SAR. An experiment is done using VV-Polarization raw data of C-band airborne SAR. The quality evaluations of compensated SAR images and uncompensated SAR images are done in the experiment. The former always performs better than the latter. After MOCO processing, azimuth ambiguity is declined, peak side lobe ratio (PSLR) effectively improves and the resolution of images is improved obviously. The result shows the validity and operability of the imaging process for airborne SAR.
\end{abstract}

\section{INTRODUCTION}

Synthetic Aperture Radar (SAR) (Raney et al. 1994, Carrara et al. 1995, and Cumming et al. 2007) can provide full-time and fullweather observation for targets, as well as the characteristics of strong penetrability, long range and high resolution. Due to these advantages, SAR has been a hot research issue in the fields of microwave imaging, opening numerous research areas and fields of applications. Comparing with space-borne SAR, airborne SAR largely overcomes the problems of long revisit time and lowresolution images. Due to the ability of flexible, accurate, and fast obtaining abundant information, airborne SAR is a significant research field of Earth Observation. In contrast to the space-borne case, airborne SAR are affected by the motion errors, since some portion of deviation from the ideal path is impossible to avoid. The ideal flight paths are straight lines, but in reality it is not the case. A small disturbance from the ideal line will dramatically deteriorating the quality of SAR images and data. Motion errors are space variant according to the data acquisition geometry, which can be split into range-variant and azimuth-variant components. Motion errors have a major effect on the signal phase and brings lower signal-noise ratio (SNR), higher azimuth ambiguity, and defocus, seriously lowering the resolution of SAR images. To get accurate echo information and radar images, it is essential to measure and compensate for nonlinear motion of antenna trajectories. Motion Compensation (MOCO) became a pivotal step in the processing of airborne SAR data. For highresolution airborne SAR systems, imaging performance is sensitive to the envelope and phase of motion errors, so a precise
MOCO is essential with the availability of high-precision Position and Orientation System (POS) data. Position and Orientation System (POS) data is applied to acquiring accuracy motion attitudes and spatial positions of antenna phase centre (APC). Since motion errors are range-variant and azimuthvariant according to the data acquisition geometry. The compensation can be split into range-variant and azimuth-variant components, and requires precise knowledge of the relative geometry between the radar and each illuminated target for every transmitted pulse. At the same time, the compensation must be a focusing strategy tailored to each illuminated target.

The MOCO procedure that accommodates trajectory deviation has been well developed in the past years. MOCO is implemented by means of phase multiplication and range delay adjustment under the range invariant assumption (John and Kirk, 1975). A two-steps MOCO method is proposed, which uses a modified chirp scaling (CS) algorithm (Raney et al. 1994, and Cumming et al. 1996) to accommodate the correction of motion errors, as well as the variations of the Doppler centroid in range and azimuth (Moreira and Huang, 1994). This method is divided into two step, range-independent compensation step and range-dependent compensation step. The first order motion compensation is processed to the range compressed data and the second order motion error correction is processed after range cell migration correction (RCMC). A generalized formulation of the extended chirp scaling (ECS) approach for high precision processing of air- and space-borne SAR data is proposed (Moreira et al. 1996). Based on the original chirp scaling function, the ECS algorithm incorporates a new azimuth scaling function and a subaperture 
approach. The two-step MOCO approach integrated in the extended chirp scaling (ECS) algorithm, which is able to correct range migration and compensate high-order phase. ECS algorithm allows motion error correction for airborne processing by means of an additional transformation into the signal domain. Recent years, many researches on MOCO have been done. Based on 3-D motion error analysis, a novel 3-D MOCO method is proposed (Xing et al. 2009). A topography MOCO method for strip-map mode based on Phase Gradient Autofocus (PFA) and ECS is developed (Zhang et al. 2014). An improved extend chirp scaling (ECS) algorithm for solving the zeros expansion problem of traditional ECS algorithm is introduced (Ye et al. 2015). An extensive investigation on RCMC analysis of one-step and twostep motion compensation is provided by establishing an accurate formula expression between the range cell migration error and the residual range-dependent phase error (Wang et al. 2016).

The whole paper is organized as follows: Section 2 gives the geometry model of the SAR imaging, analysis of motion errors, formulas derivation of MOCO, and ECS algorithm flowchart with integrated MOCO. An experiment is done using VVPolarization raw data of C-band airborne SAR in Section 3. The quality evaluations of compensated SAR images and uncompensated SAR images are done in the experiment. The former always performs better than the latter. Section 4 is the conclusion.

\section{METHOD}

\subsection{Geometry model of SAR imaging}

The SAR imaging geometry is shown in Figure 1, where the curve indicates the actual flight path, and $\mathrm{X}$-line is a virtual trajectory, conventionally nominated as the ideal path. In ideal case, the APC of the radar moves along the ideal path at a constant velocity. $\mathrm{Y}-\mathrm{Z}$ plane denotes normal plane of the ideal path. $\mathrm{H}$ is the normal flight altitude.

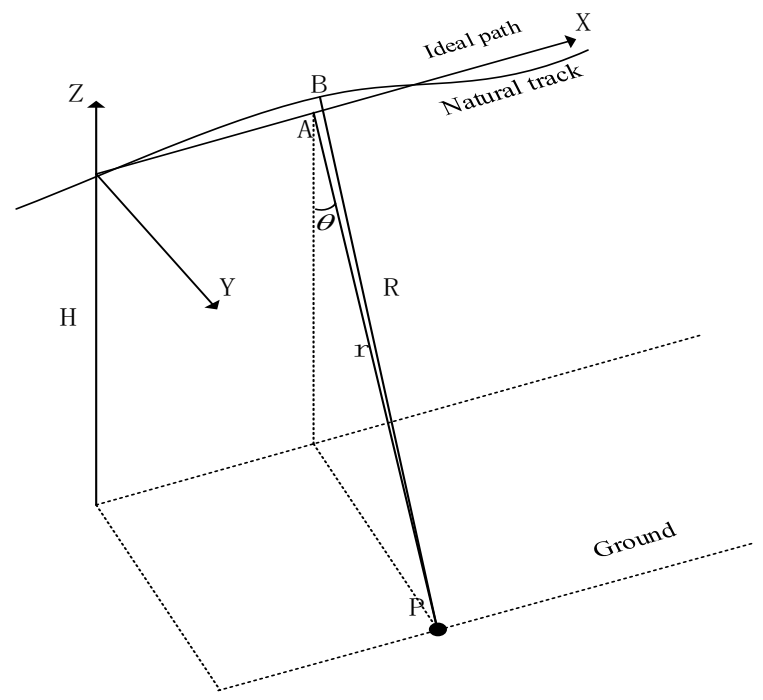

Figure 1. Geometry model of SAR imaging

\subsection{Analysis of motion error}

In Figure 1, the origin of coordinate system is the location of APC at the instant $\boldsymbol{t}=\mathbf{0}$. The slow time $\boldsymbol{t}=\boldsymbol{t}_{\boldsymbol{m}}$ is a multiple integer of the pulse repetition interval $\boldsymbol{T}_{\boldsymbol{r}}$. Assuming the average flight velocity of SAR platform is $\boldsymbol{v}$. The reference and actual positions APC position at $\boldsymbol{t}_{\boldsymbol{m}}$ are $\boldsymbol{A}\left[\boldsymbol{x}_{\boldsymbol{a}}\left(\boldsymbol{t}_{\boldsymbol{m}}\right)=\right.$ $\left.\boldsymbol{v} \boldsymbol{t}_{\boldsymbol{m}}, \mathbf{0}, \mathbf{0}\right], B\left[\boldsymbol{x}_{\boldsymbol{b}}\left(\boldsymbol{t}_{\boldsymbol{m}}\right), \boldsymbol{y}_{\boldsymbol{b}}\left(\boldsymbol{t}_{\boldsymbol{m}}\right), \boldsymbol{z}_{\boldsymbol{b}}\left(\boldsymbol{t}_{\boldsymbol{m}}\right)\right]$ respectively. The target position is $\boldsymbol{P}\left[\boldsymbol{x}_{\boldsymbol{p}}, \boldsymbol{y}_{\boldsymbol{p}}, \boldsymbol{z}_{\boldsymbol{p}}\right]$. The distance from the ideal APC position $\boldsymbol{A}$ to scatter $\boldsymbol{P}$ is

$$
R_{r e f}\left(t_{m}\right)=\sqrt{\left(x_{a}-x_{p}\right)^{2}+y_{p}^{2}+z_{p}^{2}}
$$

The distance from the natural APC position $\boldsymbol{B}$ to scatter $\boldsymbol{P}$ is

$$
R_{\text {real }}\left(t_{m}\right)=\sqrt{\left(x_{a}-x_{p}\right)^{2}+\left(y_{a}-y_{p}\right)^{2}+\left(z_{a}-z_{p}\right)^{2}}
$$

Denote the closest distance from $\boldsymbol{P}$ to ideal path $\boldsymbol{r}=$ $\sqrt{\boldsymbol{y}_{\boldsymbol{p}}^{2}+\boldsymbol{z}_{\boldsymbol{p}}^{\mathbf{2}}}$; the look angle associated with scatterer $\boldsymbol{\theta}$ Then $\boldsymbol{y}_{\boldsymbol{p}}=\boldsymbol{r} \sin \boldsymbol{\theta}, \boldsymbol{z}_{\boldsymbol{p}}=-\boldsymbol{r} \cos \boldsymbol{\theta}$. Take Taylor Expansion of $\boldsymbol{R}_{\boldsymbol{r e f}}$ and $\boldsymbol{R}_{\boldsymbol{r e a l}}$. If higher order terms are ignored, (1) can be approximated as (3) and (2) can be approximated as (4).

$$
R_{r e f}\left(t_{m}\right)=r+\frac{\left(x_{a}-x_{p}\right)^{2}}{2 r}
$$

$$
R_{\text {real }}\left(t_{m}\right)=r+\frac{\left(x_{a}-x_{p}\right)^{2}}{2 r}-y_{b} \sin \theta+z_{b} \cos \theta
$$

The displacement between the actual and nominal path is, $\left[\boldsymbol{x}_{\boldsymbol{b}}-\boldsymbol{x}_{\boldsymbol{a}}, \boldsymbol{y}_{\boldsymbol{b}}, \boldsymbol{z}_{\boldsymbol{b}}\right]$, where $\boldsymbol{y}_{\boldsymbol{b}}$ and $\boldsymbol{z}_{\boldsymbol{b}}$ denote the motion error.

\subsection{Motion compensation}

The phase error caused by the difference between $\boldsymbol{R}_{\text {ref }}$ and $\boldsymbol{R}_{\text {real }}$,

$$
\begin{aligned}
\varphi & =\frac{4 \pi}{\lambda}\left(R_{\text {ref }}-R_{\text {real }}\right) \\
& \approx \frac{4 \pi}{\lambda}\left(y_{b} \sin \theta-z_{b} \cos \theta\right)
\end{aligned}
$$

From (5), $\boldsymbol{\varphi}$ varies from $\boldsymbol{\theta} . \boldsymbol{\varphi}$ can be seen the phase compensation of one reference point $\boldsymbol{P}_{\mathbf{0}}$. The look angle of this reference point is $\boldsymbol{\theta}_{\mathbf{0}}$. The closest distance from $\boldsymbol{P}_{\mathbf{0}}$ to ideal path is $\boldsymbol{r}_{\mathbf{0}}$. Therefore the phase compensation of the first order motion compensation is

$$
\varphi_{1}=\frac{4 \pi}{\lambda}\left(R_{r e f}\left(t_{m}, r_{0}\right)-R_{r e a l}\left(t_{m}, r_{0}, \theta_{0}\right)\right)
$$

The phase compensation of the second order motion compensation is

$$
\varphi_{2}=\frac{4 \pi}{\lambda}\left(R_{r e f}\left(t_{m}, r, \theta\right)-R_{r e a l}\left(t_{m}, r, \theta\right)\right)-\varphi_{1}
$$

\subsection{ECS algorithm}

ECS algorithm uses a modified chirp scaling algorithm to accommodate the correction of motion errors, as well as the variations of the Doppler centroid in range and azimuth. ECS is computationally very efficient, since it accommodates the variations of Doppler centroid without using block processing. MOCO processing is included in the steps of ECS so that the 
image processing system is able to generate multiple compensated SAR images during imaging processing, improving the efficiency of the processing flow.

Figure 2 shows the imaging process of SAR echo data and POS data. The phase compensation is based on two hypotheses, narrow-beam antennas and horizontal imageable area.

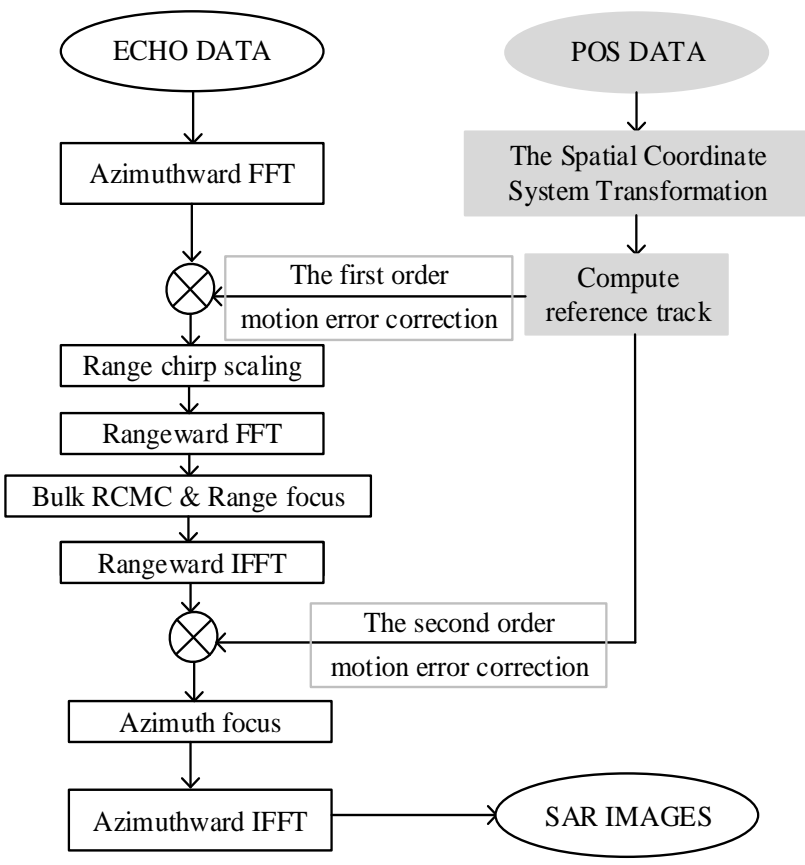

Figure 2. ECS algorithm flowchart

\section{EXPERIMENT}

ECS algorithm with integrated motion compensation and ECS algorithm without motion compensation are separately used to process VV-Polarization raw data of C-band airborne SAR in the experiment. The flight height is about $4460 \mathrm{~m}$, the velocity is $100 \mathrm{~m} / \mathrm{s}$, and the average height of object area is about $25 \mathrm{~m}$. Central incident angle is about to $52^{\circ}$. The sample interval is $0.2 \mathrm{~m}$ in range direction and $0.16 \mathrm{~m}$ in azimuth direction. The platform acquiring these images operates in side-looking mode, over Sanya of Hainan province in November, 2014.

The basic information of SAR original echo shows in Table 1.

\begin{tabular}{cc}
\hline Name & Value \\
\hline Doppler central frequency & $5.4 \mathrm{e} 9$ \\
bandwidth & $5 \mathrm{e} 8$ \\
pulse width & $2.0 \mathrm{e}-5$ \\
pulse repetition frequency & $1.25 \mathrm{e} 3$ \\
sampling rate & $7.5 \mathrm{e} 8$ \\
antenna aperture & 0.75
\end{tabular}

Table 1. The basic of information of SAR original echo

Figure 3 shows the imaging results. Figure 3(a) is the imaging result of ECS algorithm with integrated MOCO processing, and Figure 3(b) is the imaging result of ECS algorithm without MOCO processing. It is obvious that Figure 3(a) performs better than Figure 3(b) in terms of recognition of ground objects, image resolution and the retaining of target information.

Figure 4 shows quality comparisons of ground controls of two imaging process. Because of MOCO processing, the quality of mage (c) and image (e) is obviously better than image (d) and image (f). Features of objects displays more adequately, and the recognition of point targets and line targets improves significantly. Azimuth ambiguity of two ground control points declins markedly, and the focus level raises markedly.

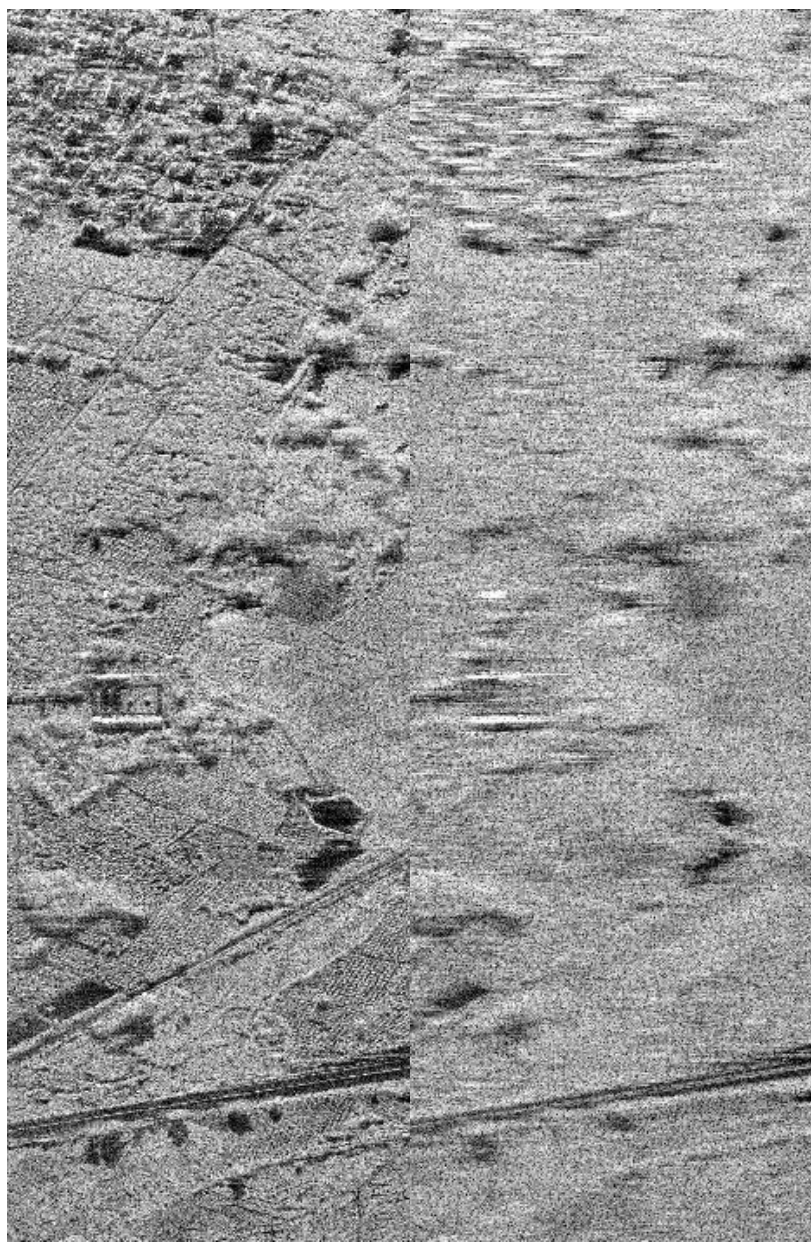

(a)

(b)

Figure 3. Imaging results of compensated and uncompensated ECS algorithm. (a) Imaging result of ECS with integrated MOCO, (b) Imaging result of ECS without MOCO.

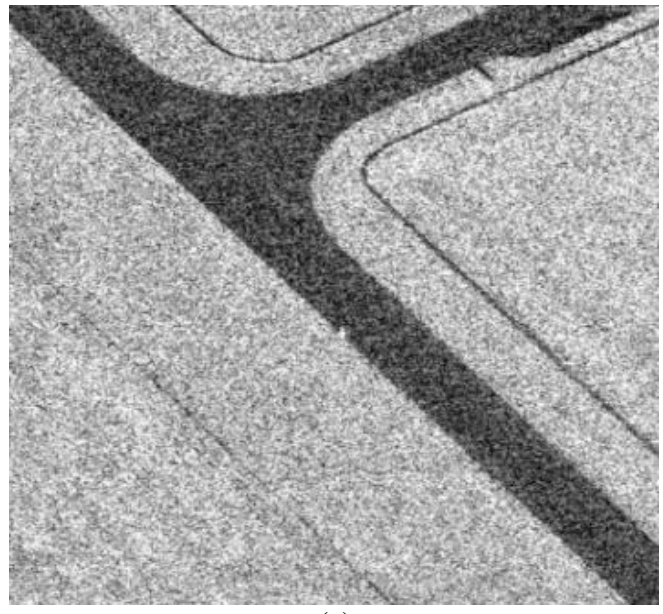

(a) 


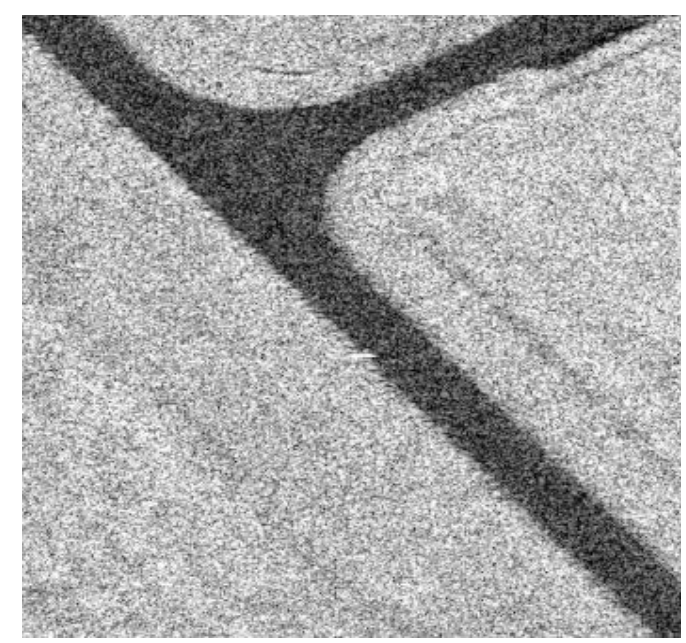

(b)

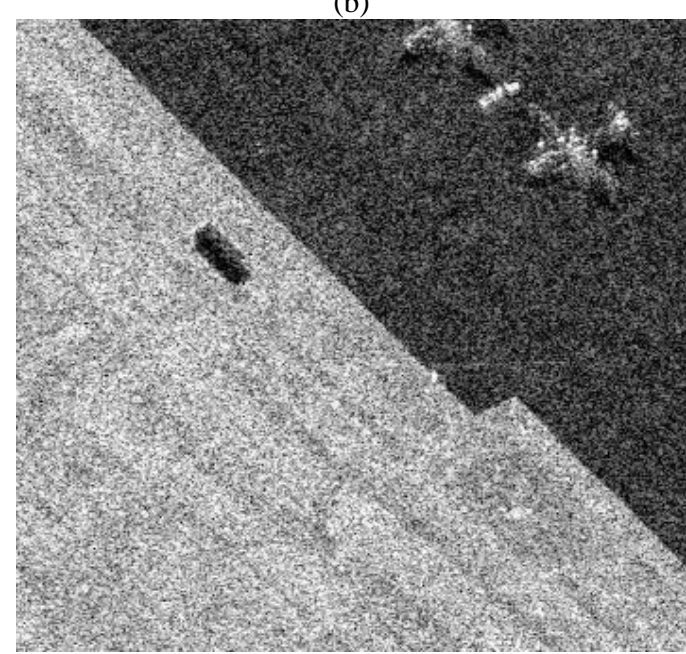

(c)

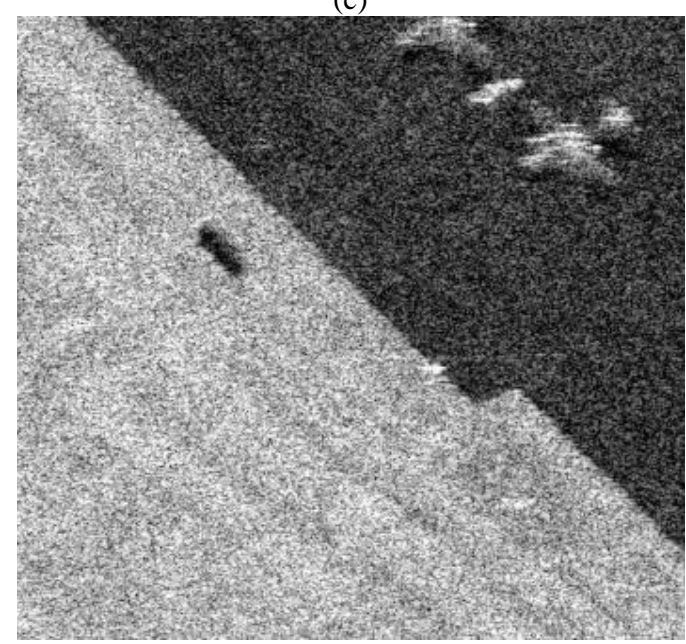

(d)

Figure 4. Comparison of ground controls of different imaging process. (a) and (c) are the results of ECS with integrated MOCO, (b) and (d) are the results of ECS without MOCO.

\section{CONCLUSION}

When using ECS imaging algorithm with integrated MOCO to process SAR data, imaging performance is sensitive to the envelope and phase of motion errors. Motion errors are able to bring lower signal-noise ratio (SNR), higher azimuth ambiguity, defocus and lower resolution. To get accurate echo information and radar images, it is essential to measure and compensate for nonlinear motion of antenna trajectories. According to the characteristics of airborne SAR data, high-precision POS data is used in a precise MOCO processing integrating with ECS algorithm. The experimental results show that the qualities of images are improved significantly after the imaging method of with integrated MOCO processing, which is a valid, useful and operative imaging process for airborne SAR.

\section{ACKNOWLEDGEMENTS (OPTIONAL)}

The authors would like to thank the anonymous reviewers for their valuable comments and suggestions. This work was supported by the key research and development plan of Hainan province under Grant NO. SY16ZY02132.

\section{REFERENCES}

Carrara, W.G., Goodman, R.S., and Majewski, R.M., 1995. Spotlight Synthetic Aperture Radar: Signal Processing Algorithms. Artech House, Boston.

Cumming, I.G., Wong, F.H., 2007. Digital processing of Synthetic Aperture Radar Data: Algorithms and Implementation. Electronic Industry Press, Beijing.

Cumming I.D., Ito, M.R., 1996. A chirp scaling approach for processing squint model SAR data. IEEE Trans. Aerospace Electronic Syst. 32(1), 121-133.

John, C., Kirk, JR., 1975. Motion compensation for Synthetic Aperture Radar. IEEE Trans. AES-i1(3), pp.338-348.

Moreira, A., Huang Y.H., 1994. Airbome SAR processing of highly squinted data using a chirp scaling approach with integrated motion compensation. IEEE Trans. 32(5).

Moreira, A., Mittermayer, J., and Scheiber, R., 1996. Extended Chirp Scaling algorithm for air- and spaceborne SAR data processing in Stripmap and ScanSAR imaging modes. IEEE Trans. 34(5).

Raney, R.K., Runge, H., Bamler, R., Cumming, I.G., and Wong, F.H., 1994. Precision SAR processing using chirp scaling. IEEE Trans. GRS 32 (4), pp.786-799.

Wang. G.Y., Zhang, L., Li, J., and Hu, Q.R., 2016. Range cell migration correction analysis of one-step and two-step motion compensation for millimeter-wave airborne SAR imaging. EURASIP Journal on Advances in Signal Processing, 2016(1), pp.1-11.

Xing, M.D., Jiang, X.W., Wu, R.B., Zhou, F., and Bao, Z., 2009. Motion Compensation for UAV SAR based on raw radar data. IEEE Trans. 47(8).

Ye, X.M., Zhang, G.F., and Hu, X.G., 2015. An improved ECS algorithm for missile-borne SAR based on scaled Fourier transform. Optik-International Journal for Light and Electron Optics, 126(18), pp.1633-1639.

Zhang, L., Liu, Y., and Yang, L., 2014. Motion compensation using substrip PGA. Journal of electronics(China), 31(4). 